Journal of Computer Science 8 (4): 556-563, 2012

ISSN 1549-3636

(C) 2012 Science Publications

\title{
Third Order of Automatic Restoration Scheme Offered in Fiber ToThe Home-Passive Optical Network through Customer Access Protection Unit
}

\author{
Mohammad Syuhaimi Ab-Rahman, \\ Siti Asma Che Aziz and Kasmiran Jumari \\ Department of Electrical, Electronics and System Engineering, \\ Faculty of Engineering and Built Environmental, \\ University Kebangsaan Malaysia, 43600 UKM Bangi, Selangor, Malaysia
}

\begin{abstract}
Problem statement: This study highlight on restoration scheme proposed against failure in working line at the drop region for Fiber To The Home (FTTH) with a Passive Optical Network (PON). Whereas PON is a system that brings optical fiber cable and signals all or most of the way to the end user. In this study we highlighted the issue on survivability scheme against failure which is focused scattered residence architectures. Approach: Two type of restoration scheme is proposed by means of linear protection (tree) and migrate protection (ring). Our proposal scheme is inexpensive and applicable to any residence architecture (ordered placement and scattered placement). The advantage of this scheme is the failure at fiber line can be recovered until two levels to make sure the optic signal flow continuously. FTTH based network design is simulated by using opti system 7.0 in order to investigate the power output and BER performance at each node in the tree and ring protection scheme in scattered placement to prove the system feasibility. Results: Computation of Channel Dissipation on Each Breakdown Condition has give the insertion for $16.4 \mathrm{~dB}$ (normal condition), 18.8 $\mathrm{dB}$ (failure order 1), $21.2 \mathrm{~dB}$ (failure order 2) and $23.6 \mathrm{~dB}$ (failure order 3). Simulation was carried out to study the maximum distance that can be achieved for signaling path in two situations which are; in normal and breakdown condition with the restoration scheme. The sensitivity that used in analysis to study the maximum fiber length is $-25,-30$ and $-35 \mathrm{dBm}$. The result shows output power that linearly decrease as the fiber length is increased; for four breakdown condition in this protection network. The highest output power achieved by normal condition at each increase in distance compared to the output power at level 3 breakdown. In addition, the accessibility of maximum distance is dependent on the sensitivity used. In order to achieve a high fiber length of more than 20 $\mathrm{km}^{-1}$ at third level breakdown, the proposed sensitivity should exceed $-33 \mathrm{dBm}$. It is based on output power at third level breakdown that recorded at $-32.502 \mathrm{dBm}(1490 \mathrm{~nm})$ for orderly placement of this protection network. Conclusion: This study suggests the migration of tree to ring topology to enable the signal flow continuously in the case of failure occurs specifically in random or scattered placement topology. Our proposal is the first reported up to this time in which the upstream signal flows in anticlockwise in ring topology when the restoration scheme activated.
\end{abstract}

Key words: Passive Optical Network (PON), Voice over Internet Protocol (VoIP), Optical Line Terminal (OLT), Client Access Protection Unit (CAPU)

\section{INTRODUCTION}

From Wikipedia, the meaning of Fiber To The X (FTTX) is a network architecture that uses fiber optic to replace a part of copper loop that is commonly used in telecommunications network system in nowadays. Four technologies which related to this fiber loop are (Wikipedia):

- $\quad$ Fibre To The Node/neighborhood (FTTN)/Fiber To The Cabinet (FTTCab)

- Fibre To The Curb (FTTC)/Fibre To The Kerb(FTTK)

Correspending Author: Mohammad Syuhaimi Ab-Rahman, Department of Electrical, Electronics and System Engineering, Faculty of Engineering and Built Environmental University Kebangsaan Malaysia, 43600 UKM Bangi, Selangor, Malaysia 
- $\quad$ Fibre To The Building (FTTB) Fibre To The Home (FTTH)

In this project, we highlight the deployment of consolidation of voice, data and video, as well as functioning like junction box in a residence or business buildings. Sometimes it also called as Fiber To The Building (FTTB). This will open up a space for improvement of internet, the use of continuous video and other applications. Internet is using fiber optic cables that capable to deliver the effective and good bandwidths. This capability causing an Internet to be the main source of network technology that can be used at home and involve in business matter. However, the data entry $\log$ for this network has a limited capacity. This will affect the creation of an ideology to enhance the existing technology that requires a greater bandwidth; it is FTTH. The current issue on FTTH deployment is the survivability. Survivability is the capability of the system to preserve the service in the case of component breakdown sech as devices and optical cable (Ab-Rahman et al., 2011). Noted that survivability in FTTH network is an important issue because it has been discussed in optical network by earlier studies (Yeh and Chi, 2008; Aziz et al., 2009; Premadi et al., 2010 Hossain et al., 2005; Zhao et al., 2005). The concept has also been extended in WDMFTTH network (Han et al., 2007; Chan et al., 2003). The main key of the solution is the effective monitoring solution to determine the network health $(\mathrm{Ng}$ et al., 2010). The extension of optical solution up to customer devices has also been reported in many publication such as Visible Light Communication (VLC) and POF network for home networking (Rajbhandari et al., 2011). Therefore the bandwidth is insignificant problem to increase the user application which require high bandwidth demand.

Fiber optic cable is made from glass fiber where it can carry data that accelerate the data transmission which is more that 2.5 Gigabits per second (Gbps). FTTH services usually offer different speed and it is closely related to the price. FTTH that has expensive value is having the strength to speed up the data transfer more that 100 Mbps which means, 66 times faster than a regular DSL. Although the multiplexing method has set the digital signals to be send through an existing channel, but this fiber optic wires can carry these signals with high speed and unlimited bandwidth. FTTH network is an optimum bandwidth, practical and capable to be a telecommunication solution that used fiber optic cable and optical electronics where it can deliver a various types of broadband services. It allows very rapid data transmission to homes and offices that requires the service to provide IP-based services such as IPTV and High Speed Broad Band (HSBB). With this capability, users will be satisfied with the use of IPTV, Video-on-Demand entertainment and games. Voice over Internet Protocol (VoIP) services and data transmission is through a fiber facility that enables a broadband connection (Abdul-Rahman and Wang, 2010).

Structure: Before we look further on a structure of FTTH, we need to know the basic information for data transmission system. Figure 1 shows a diagram of information system in general. The data that obtained from streamer, routers and PABX S12 PSTN switches will be sent to network management system. Then the data will be sent to Direct PON and will determine to be distributed according to the speed of either going to FTTB, FTTH or business access media.

In structure of FTTH network as in Fig. 2, three key components that involved are:

- Central Office (CO)/Optical Line Terminal (OLT)

- Splitter

- Optical network terminal

The data was sent to the central office (exchange). After the data processing, it will be distributed at splitter in accordance with the speed and then it will be sent to Optical Network Terminal at home (consumer), as in Fig. 3.

Figure 3 the structure of Optical Line Termination (OLT) (Head-end) consists of Optical Distribution Network (ODN) and Optical Network Unit (ONU) where 1:4 ratio is used for ONU to the home (consumer). Cable in use is the optical fiber.

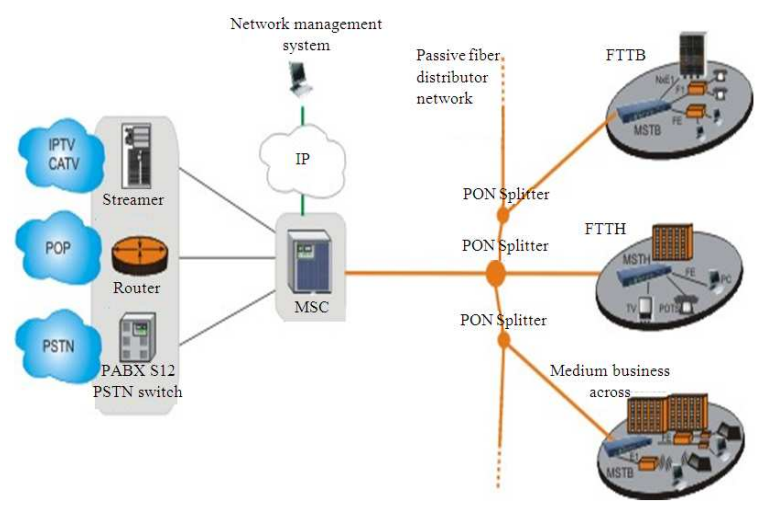

Fig. 1: Diagram of information system (FTTH, 2005) 


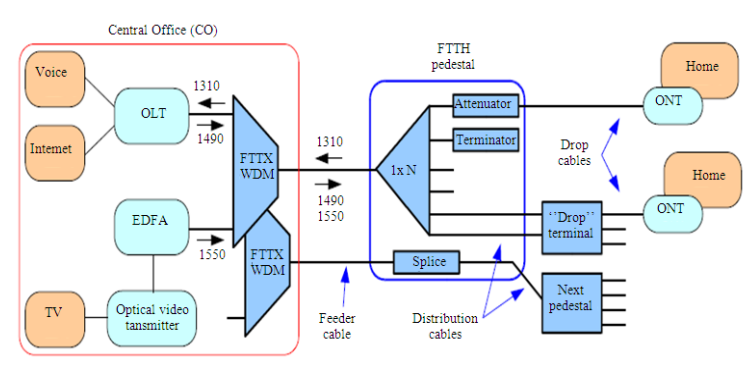

Fig. 2:Relationship between three key components (FTTH, 2005)

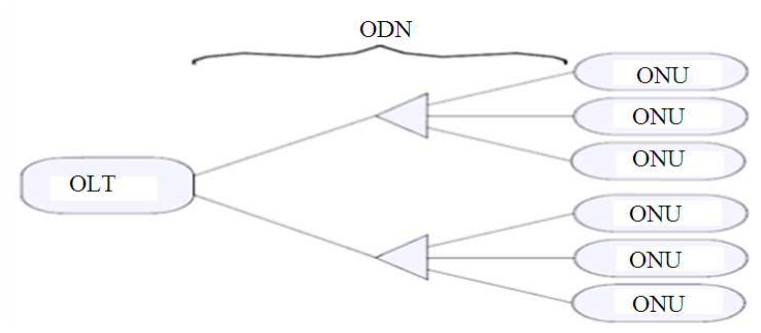

Fig. 3: The structure of Optical Line Termination (OLT) (Head-end) consists of Optical Distribution Network (ODN) and Optical Network Unit (ONU) where 1:4 ratio is used for ONU to the home (consumer). Cable in use is the optical fiber (FTTH, 2005)

\section{MATERIALS AND METHODS}

Fiber optic: Figure 4 shows a cross section of basic optical fiber channel. In optical fiber applications, the data (in form of voltage) is converted to light before channeled into the optical fiber channel. Before doing that process, some of the light channel will be set, so that it will be in a good reflection. A cladding is an outline of fiber optic that functions to reflect the data in form of light at the core or a data flow. Data will move in the speed of light which is $3.8 \times 0 \mathrm{~m} \mathrm{sec}^{-1}$. The ability of optical fiber to transmit data with minimum noise interference makes it as a perfect medium to transmit data.

Optical line terminal: Optical Line Terminal (OLT) is a destination data for FTTH channel. In other words, it functions as a terminator for optical fiber network in central office. OLT is usually used in Passive Optical Network (PON). This is because; the function is suitable for data transmission of fiber optic in various forms, which are video, audio, streaming, internet.

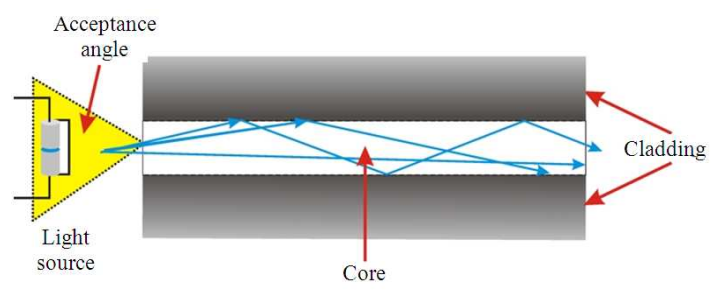

Fig. 4: Cross section of basic optical fiber channel (Wikipedia)

The ability of OLT to control the data flow is very good. The temperature effect is the main contributors to the system interference in the box (Aleksic and Lovric, 2011).

Optical network Unit (ONU/ONT): Although these tools have two different names but its function is still the same. The deferential form of ONU and ONT is due to; one is from IEEE term and the other one is from Communication term. ONU/ONT is a tool that convert optical signal into electrical signal. In other words, these tools translate the optical input in form of light into electrical signal to be encoded by receiving end which is end user. ONU/ONT also can convert the optical signal in forms of video, audio, streaming, internet before being used. Hence, this tool is very important in FTTH application.

Additional prototype: To realize the proposal of high survivability FTTH network we have introduced two new prototypes which respectively to control and activate the restoration scheme. The devices are built based on electronic and optical integration design and was patented by University Kebangsaan Malaysia with commercialized name Access Control System (ACS) and Customer Access Protection Unit (CAPU), as shown in Fig. 5. The control system is used to divert the traffic to the alternative path and the same time to sense the breakdown at the specific line. The second devices support the restoration scheme by shifting the receiver to the specific line where the signal is coming.

Latest technology of FTTH: In FTTH, there are two commonly used technologies which are Point to Point (PTP) and Passive Optical Network (PON). PTP technology is a technology that uses single fiber from one point to another point without any additional equipment while $\mathrm{PON}$ is a technology that uses passive component only. There are various PON technologies that have evolved from one version to another advanced version. The first PON technology that was introduced in 1995 is ATM-PON or better known as APON. 

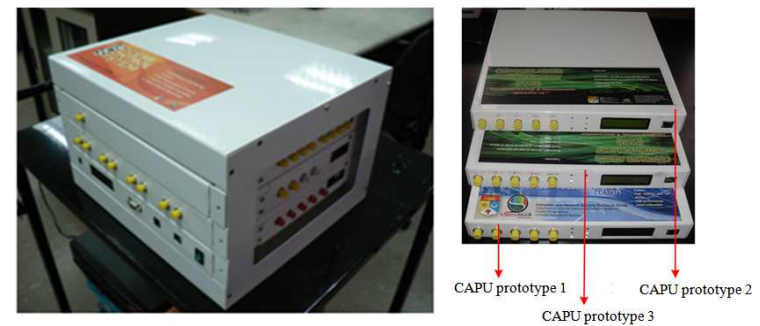

Fig. 5: Two new prototypes have been developed to support the restoration scheme offered by FTTHPON

Table 1: Comparison between PON

\begin{tabular}{llll}
\hline Characteristic & EPON & BPON GPON & \\
\hline $\begin{array}{l}\text { Standard } \\
\text { G.984 }\end{array}$ & IEEE $802.3 \mathrm{ah}$ & ITU-T G.983 & ITU-T \\
$\begin{array}{l}\text { Protocol } \\
\text { TDM }\end{array}$ & Ethernet & ATM & Ethernet, \\
Rates & DS $=1000 \mathrm{Mbps}$ & & \\
US $=1000 \mathrm{Mbps}$ & DS $=622 \mathrm{Mbps}$ & & \\
US $=155 \mathrm{Mbps}$ & DS $=2488 \mathrm{Mbps}$ & & \\
US $=1244 \mathrm{Mbps}$ & & & \\
Span $(\mathrm{km})$ & 10 & 20 & 20 \\
Split ratio & 16 until 32 & 32 & 32 until 64 \\
\hline
\end{tabular}

In 1998, Broadband-PON or BPON was introduced. Then, this technology continues to grow until the Gigabyte-PON or GPON was introduced in 2003. Ethernet-PON or EPON comes next where it has been introduced in 2004. Wavelength Division Multiplexing (WDMPON) is one of the latest technologies in the implementation of FTTH but it is still in research level. Wavelength Division Multiplexing (WDM) is assumed to be the key technology for bringing about more bandwidth to the customer in a later stage of network evolution and first using coarse channel spacing. If an adequate demand for bandwidth as well as for protection is predictable it is useful to switch over to WDM fiber rings with optical add-drop multiplexing (Langer et al., 2004). Figure 6 below shows basic structure in WDMPON. While Table 1 shows some differences between PON that has been stated earlier.

FTTH related issue: In 2011, Japan has the highest percentage in the use of FTTH technology. The rapid developments in their country have contributed to the growth rate in implementation of FTTH. Policies in Japan, such as e-Japan also led to this growth. Same goes in Korea, where the government policies that been enforced such as e-Korean has helped in development of FTTH at their country.

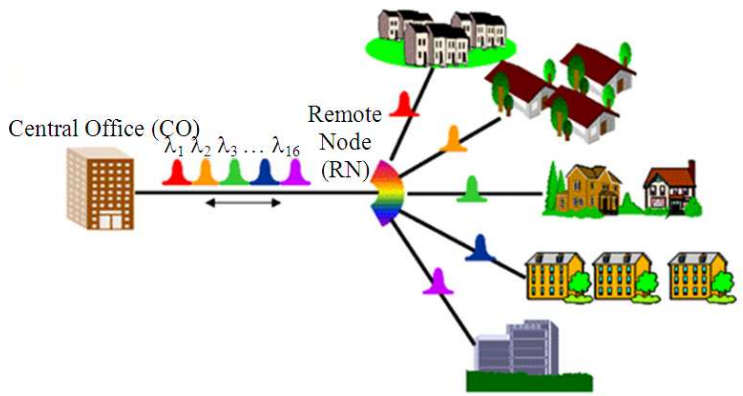

Fig. 6: Basic structure in WDMPON Wikipedia

On the other hand, in 2002, the highest cost that involves of FTTH installation and usage has reached until \$5500. However the cost is decreasing, where in 2009 , the cost has been reduced to $\$ 500$. Therefore, it is not impossible if someday FTTH can be implemented in Malaysia if the cost continues to decrease up to a level that affordable by people of Malaysia.

Comparison between FTTH and VDSL: There are several aspects that will be a point of comparison between FTTH and Very high bit-rate Digital Line Subscriber (VDSL). The first one is; in terms of construction basic materials where FTTH uses fiber as basic medium while VDSL uses copper. Fiber uses laser light to carry information and its signal can travel as far as 15 miles (over $25 \mathrm{~km}^{-1}$ ). VDSL uses twistedpair cable that made from copper which also use a radio frequency carrier on the voice signal.

In terms of speed, the FTTH can reach $10 \mathrm{Gbps}$ but for home users, the speed is limited to only $10 \mathrm{Mbps}$. For VDSL, it could achieve more than $200 \mathrm{Mbps}$ but only for 750 feet $(228.6 \mathrm{~m})$; while for a distance of 1 mile $(1.6$ $\mathrm{km}^{-1}$ ), it only capable of achieving up to $30 \mathrm{Mbps}$. Here we can see that VDSL has a relatively limited speed compared to FTTH because its speed is inversely proportional to the distance. An operating range can also be a point of comparison. FTTH can operate for longer distances which are within $10-20 \mathrm{~km}^{-1}$. VDSL only able to operate in a shorter distance which is within $5 \mathrm{~km}^{-1}$ only. Therefore, FTTH is more suitable to be used for remote operating system.

FTTH requires a higher cost for maintenance because of need to plant the fiber in the ground and prepare for the individual core, while VDSL is quite cheap (Rejeb et al., 2010). However, in the current economic conditions, labor costs required to be consider. To reduce the maintenance cost of FTTH, a fiber distribution box has installed on the existing cable. This method is said to be faster and cheaper than fiber planting in the ground. 


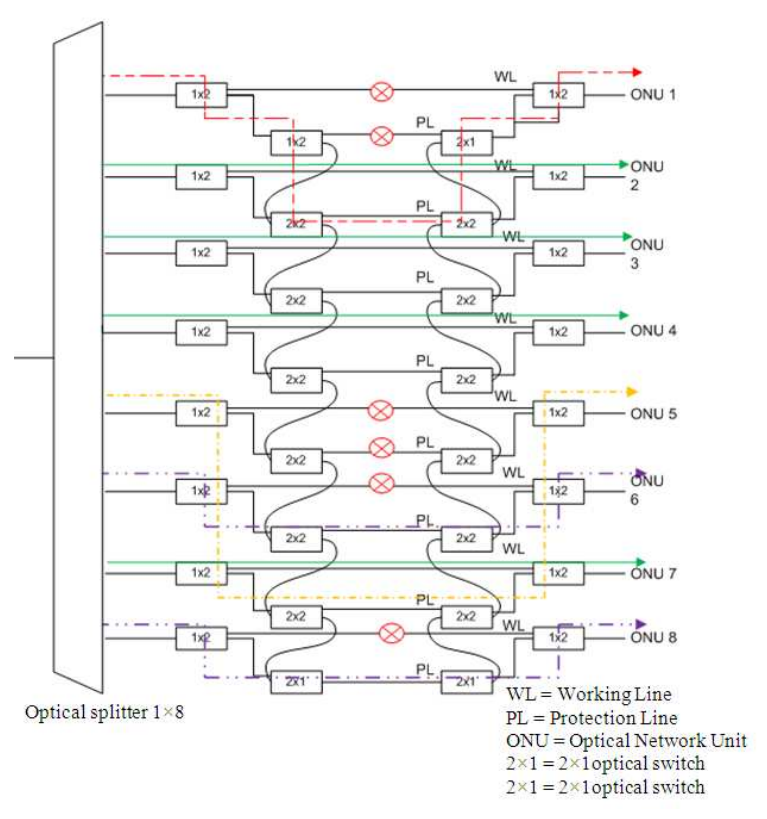

Fig. 7: The protection mechanism against breakdown with its recovery conditio

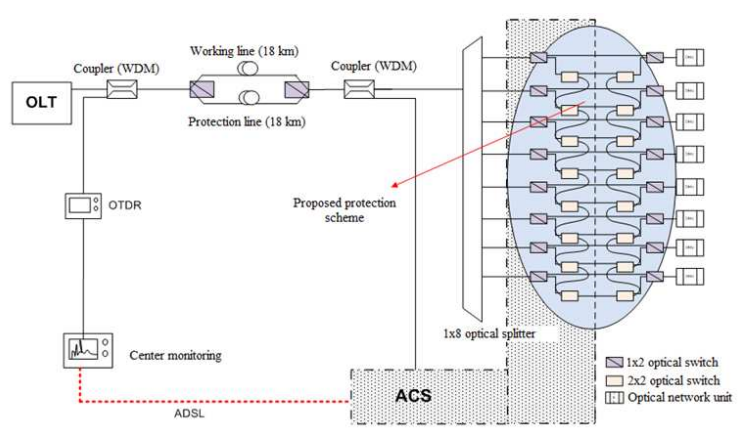

Fig. 8: Intelligent FTTH network design with protection schemes in distribution part

According to RVA Market Research source, $89 \%$ of users were satisfied with the services provided by FTTH, while only about $63 \%$ for VDSL users. It can be concluded that the services provided by FTTH is better than VDSL.

Protection design: Figure 7 shows a proposal of protection schematic mechanism for 8 users. Each protection line will be connected with neighboring protection line by forming a zig zag pattern. Line with green arrows in earlier figure shows the optical signal through line will work when there is no damage reported. Proposal for the damage recovery will be covered for up to three levels of damage which sequentially in the line. Figure 8 shows a proposed protection mechanism to be applied in development of intelligent FTTH at the research laboratory. This proposed mechanism will be placed on the distribution in network before the optical signal is sent to users as an alternative protection from any breakdown that occurs at line work. Every optical switch will be controlled by ACS. Development tools for the protection switch, known as Client Access Protection Unit (CAPU) which will be placed on the customer part will be discussed later.

\section{RESULTS AND DISCUSSION}

Computation of channel dissipation on each breakdown condition: This analysis aims to compute the channel dissipation that has been provided by protection scheme on the developing systematic placement. Among the components that involved in the proposed linear protection network is optical switch and $1 \times 8$ optical splitter. Following are the calculations of channel dissipation for signal path on $1490 \mathrm{~nm}$ which is in normal condition and line breakdown:

Normal signaling path $=\left(20 \mathrm{~km}^{-1}\right.$ optical fiber $)$ + (1xoptical splitter)

$+(2 \times$ optical switch $)=\left(20 \times \mathrm{km}^{-1} \times 0.25 \mathrm{~dB} \mathrm{~km}^{-1}\right)$

$+(1 \times 9.0 \mathrm{~dB})$

$+(2 \times 1.2 \mathrm{~dB})=16.4 \mathrm{~dB}$

Breakdown signaling path at level:

$1=\left(20 \mathrm{~km}^{-1}\right.$ optical fiber $)+(1 \times$ optical splitter $)$

$+(4 \times$ optical switch $)=\left(20 \mathrm{~km}^{-1} 0.25 \mathrm{~dB} \mathrm{~km}^{-1}\right)$

$+(1 \times 9.0 \mathrm{~dB})+(4 \times 1.2 \mathrm{~dB})=18.8 \mathrm{~dB}$

Breakdown signaling path at level:

$2=\left(20 \mathrm{~km}^{-1}\right.$ optical fiber $)+(1 \times$ optical splitter $)$

$+(6 \times$ optical switch $)=\left(20 \mathrm{~km}^{-1} \times 0.25 \mathrm{~dB} \mathrm{~km}^{-1}\right)$

$+(1 \times 9.0 \mathrm{~dB})+(6 \times 1.2 \mathrm{~dB})=21.2 \mathrm{~dB}$

Breakdown signaling path at level:

$3=\left(20 \mathrm{~km}^{-1}\right.$ optical fiber $)+(1 \times$ optical splitter $)$

$+(8 \times$ optical switch $)=\left(20 \mathrm{~km}^{-1} \times 0.25 \mathrm{~dB} \mathrm{~km}^{-1}\right)$

$+(1 \times 9.0 \mathrm{~dB})+(8 \times 1.2 \mathrm{~dB})=23.6 \mathrm{~dB}$.

Total distance of protection scheme network: Simulation was carried out to study the maximum 
distance that can be achieved for signaling path in two situations which are; in normal and breakdown condition with the restoration scheme. The sensitivity that used in analysis to study the maximum fiber length is $-25-30$ and $-35 \mathrm{dBm}$. Figure 9 shows the output power that linearly decrease as the fiber length is increased; for four breakdown condition in this protection network. The highest output power achieved by normal condition at each increase in distance compared to the output power at level 3 breakdown.

In addition, the accessibility of maximum distance is dependent on the sensitivity used. In order to achieve a high fiber length of more than $20 \mathrm{~km}^{-1}$ at third level breakdown, the proposed sensitivity should exceed -33 $\mathrm{dBm}$. It is based on output power at third level breakdown that recorded at $-32.502 \mathrm{dBm}(1490 \mathrm{~nm})$ for orderly placement of this protection network. To study the effect of power on fiber length accessibility at particular sensitivity, thus a protection network simulation with its recovery being conducted when fiber length is ncreasing. Figure 10 shows the fiber length (distance) that can be achieved in a sensitivity of $-25 \mathrm{dBm}$. The maximum distance was found to vary according to situation breakdown in which the use of optical switches in each case is different. Found that, on the sensitivity of $-25 \mathrm{dBm}$ is not appropriate to be conducted because the signal at third level breakdown not able to achieve the $\mathrm{Q}$ factor of 6 . This is because the signal is too low when too much power dissipation in that signal.

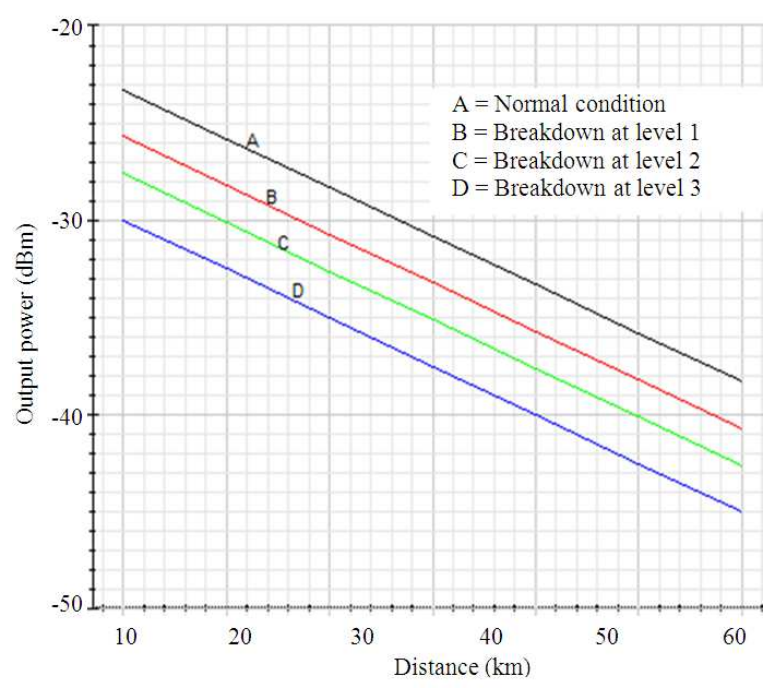

Fig. 9: Output power against fiber length $(\mathrm{km})$ for all condition
In addition, data will be difficult to be separated from noise and make the quality factor be too low. Thus, the sensitivity should be further improved to obtain a good performance at the receiver for all breakdown situations.

If the sensitivity is increased to $-30 \mathrm{dBm}$, the result is then shown as in Fig. 11. From that figure, it shows the impact of high $\mathrm{Q}$ factor at sensitivity of $30 \mathrm{dBm}$ compared with sensitivity of $-25 \mathrm{dBm}$ that has been carried out previously.

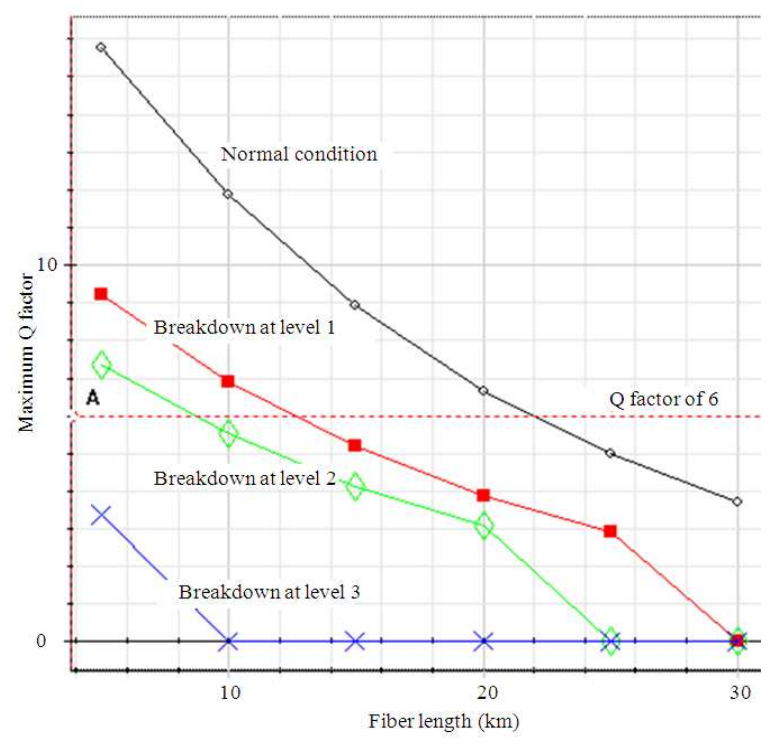

Fig. 10: Effect of $Q$ factor of fiber length $(\mathrm{km})$ for each breakdown situation

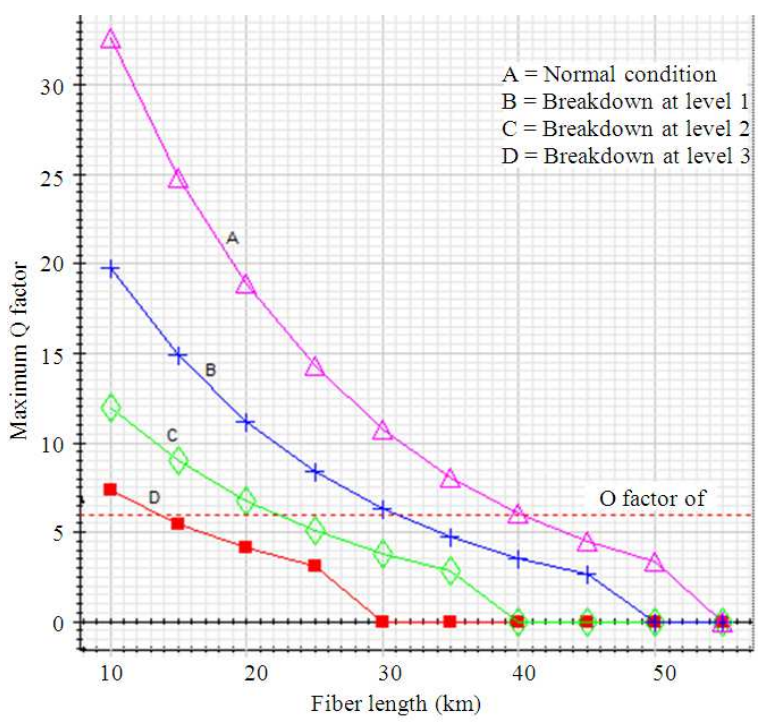

Fig. 11: Maximum $Q$ factor against fiber length $(\mathrm{km})$ at sensitivity of $-30 \mathrm{dBm}$ 


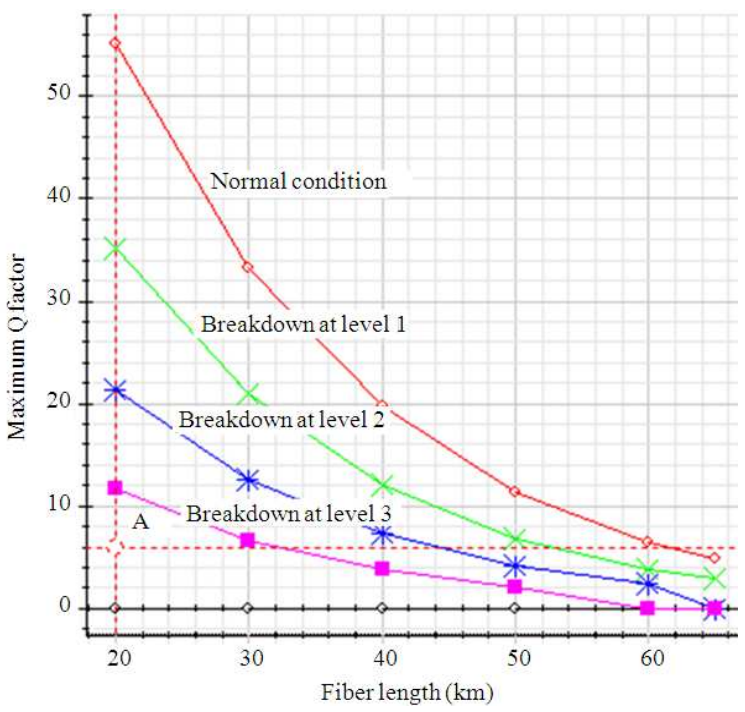

Fig. 12: Maximum $Q$ factor against fiber length at sensitivity of $-35 \mathrm{dBm}$

The maximum distance at third level of breakdown is only $13.3 \mathrm{~km}^{-1}$ compared to $40 \mathrm{~km}^{-1}$, which is at normal conditions without breakdown? However, a distance of $13.3 \mathrm{~km}^{-1}$ is still low thus the sensitivity will be further enhanced in order to achieve greater distance. As sensitivity is increased at $-35 \mathrm{dBm}$, then the whole level of breakdown will reach the $\mathrm{Q}$ factor at least 6 . Figure 12 shows an exponentially decrease for a signal in various situations where fiber length is increased to $65 \mathrm{~km}^{-1}$ and the maximum distance achieved is $60.5 \mathrm{~km}^{-1}$ for signal at normal conditions without breakdown. For a situation at third level of breakdown, the maximum distance that can be achieved in the sensitivity is only $32.4 \mathrm{~km}^{-1}$.

\section{CONCLUSION}

In the future, FTTH technology will bring benefits to consumers that concerned with bandwidth capacity, reliability, security and scalability to the single optical fiber cable for all Internet Protocol (IP) based in services simultaneously. We pointed out that FTTH is best used in the current world telecommunication system because it can bring a faster broadband consolidation services for voices, data and video.

Furthermore, FTTH network has an optimum bandwidth, practical and capable to be telecommunication solutions using optical fiber cables and optical electronics which will deliver various broadband solutions that rapidly developing nowadays.

\section{REFERENCES}

Abdul-Rahman, H. and C. Wang, 2010. Critical Retrospect on Conventional and Luminescent Solar Concentration Devices. Am. J. Environ. Sci., 6: 428-437. DOI: 10.3844/ajessp.2010.428.437

Ab-Rahman, M.S., M. Tanra, S.C. Rosli, B. Ng and A. Baharudin et al., 2011. Analysis of components failure, malfunction effect and prevention technique in customer access Network FTTHPON. J. Applied Sci., 11: 201-211.

Aleksic, S. and A. Lovric, 2011. Energy consumption and environmental implications of wired access networks. Am. J. Eng. Applied Sci., 4: 531-539. DOI: 10.3844/ajeassp.2011.531.539

Aziz, S.A.C., M.S. Ab-Rahman and K. Jumari, 2009 Customer access protection unit for survivable FTTH network. Proceedings of the International Conference Space Science Communication, Oct. 26-27, IEEE Xplore Press, Nigeria Sembilan, pp: 71-73. DOI: 10.1109/ICONSPACE.2009.5352668

Chan, T.K., C.K. Chan, L.K. Chen and F. Tong, 2003. A self-protected architecture for wavelengthdivision-multiplexed passive optical networks. IEEE Photon. Technol. Lett., 15: 1660-1662. DOI: 10.1109/LPT.2003.818657

Han, K.E., S.H. Shim, B.J. Oh, L.M. Peng and Y.C. Kim, 2007. Hybrid Protection Architecture against Multipoint Failure in WDM-PON. Proceedings of the 9th International Conference on Advanced Communication Technology, Feb. 12-14, IEEE Xplore Press, Gangwon-Do, pp: 1385-1390. DOI: 10.1109/ICACT.2007.358615

Hossain, D., H. Erkan, R. Dorsinville, M. Ali and S. Shami et al., 2005. Protection for a Ring-Based EPON Architecture. Proceedings of the 2nd International Conference on Broadband Networks, Oct. 7-7, IEEE Xplore Press, Boston, MA., pp: 1548-1553. DOI: 10.1109/ICBN.2005.1589790

Langer, K.D., J., Grubor and K. Habel, 2004. Promising evolution paths for passive optical access networks. Proceedings of 2004 6th International Conference on Transparent Optical Networks, Jul. 4-8, IEEE Xplore Press, pp: 202-207. DOI: 10.1109/ICTON.2004.1360276

Ng, B., M.S. Ab-Rahman and A. Premadi, 2010. Development of monitoring system for FTTH-PON using combined ACS and santad. Int. J. Commun. Syst., 23: 429-446. DOI: 10.1002/dac.1078

Premadi, A., B.C. Ng, M.S. Ab-Rahman and K. Jumari, 2010. Access network survivability: An architecture approach for monitoring, protection and restoration in FTTH application. Ann. Telecommun., 65: 263-269. DOI: 10.1007/s12243009-0155-5 
Rajbhandari, S., Z. Ghassemlooy and M. Angelova, 2011. The performance of a dual header pulse interval modulation in the presence of artificial light interferences in an indoor optical wireless communications channel with wavelet denoising. Am. J. Eng. Applied Sci., 4: 513-519. DOI: 10.3844/ajeassp.2011.513.519

Rejeb, R., M.S. Leeson, C.M. Machuca and I. Tomkos, 2010. Control and management issues in all-optical networks. J. Netw., 5: 132-139. DOI: 10.4304/jnw.5.2.132-139
Yeh, C.H. and S. Chi, 2008. Self-protection against fiber fault for ring-based power-splitting passive optical networks. Optical Eng.-Bell.-Int. Soc. Optical Eng., 47: 020501-020501.

Zhao, X., X. Chen and X. Fu, 2005. A novel protection switching scheme for PONs with ring plus tree topology. Proc. SPIE, 6022: 60223H-60223H. DOI: $10.1117 / 12.636269$ 